\title{
TSAR : un modèle mono-dimensionnel de simulation des évolutions des fonds alluvionnaires des rivières
}

\author{
E. Ben Slama, S. Péron \\ (EDF/LNH) \\ P. Belleudy
}

(LHF)

G. Rouas

(STCPMVN)

\section{Introduction}

Fruit d'une collaboration entre plusieurs partenaires [1], le projet TSAR a pour objectif la réalisation d'un modèle numérique mono-dimensionnel performant et à vocation industrielle pour l'étude des évolutions des lits fluviaux.

Cet outil vise notamment la simulation, à des échelles de temps variables, des processus complexes de dépôtérosion et de transport par charriage et suspension de sédiments non cohésifs en granulométrie étendue tels qu'ils se produisent dans des rivières de plaines soumises à des écoulements subcritiques.

Le projet s'est déroulé selon trois phases distinctes :

- la première était consacrée à l'étude théorique et expérimentale du transport solide par charriage des sédiments non cohésifs du type sable et gravier,

- la seconde phase qui fait l'objet de cet article a porté sur le développement et la validation d'une première version du code TSAR,

- la troisième phase en cours de réalisation permettra l'intégration et le fouplage avec le charriage d'un modèle de transport des sédiments en suspension par convectiondiffusion.

Après un bref rappel des principaux résultats obtenus au cours des études expérimentales [2], [3], nous présentons les spécifications du code TSAR dans sa première version et quelques cas tests de validation qui prouvent d'ores et déjà l'étendue de son champ d'application.

Parmi les options de modélisation adoptées dans le cadre du développement de TSAR, nous mettons l'accent sur le choix d'une méthode numérique découplée qui enchaîne la résolution de méthodes adaptées aux systèmes d'équations liquides et solides. Cette méthode, qui s'avère efficace quant à la représentation physique des phénomènes, doit favoriser en particulier le couplage des modules sédimentologiques développés dans le cadre du projet avec différents modules hydrodynamiques existants. Elle est, à ce titre, conforme à l'une des orientations principales du projet, à savoir la modularité du code, qui permettra d'en faciliter la maintenance et les futurs développements.

TSAR : a monodimensional model for simulating the evolution of rivers alluvial beds

The code TSAR aims at predicting short and medium-term evolutions of rivers beds subject to drifting or varied grain size material suspension transport. It is based on a representation of the straight section of the flow, which makes easier banks evolution processing. The bed is modelled by stratums caracterized by their thickness and grain size distribution.

This paper shows the main conclusions of the first tests of the code. 


\section{Rappel des principaux résultats expérimentaux}

Réalisés dans un canal rectiligne de $40 \mathrm{~m}$ de longueur utile et de $0,4 \mathrm{~m}$ de largeur, les essais ont porté sur l'étude en régime permanent du transport par charriage, sans recirculation, de matériaux sableux ou graveleux en granulométrie uniforme ou étendue.

La mesure en continu des fonds initialement plats et de la surface libre de l'écoulement sur l'ensemble du canal a tout d'abord permis de suivre l'évolution des formes du lit au cours du temps. L'apparition et le déplacement de dunes et rides, de formes variables selon la composition initiale du lit et la valeur des débits liquides ont mis en évidence l'importance du rôle joué par la rugosité qui conditionne la force tractrice et donc le transport solide [3].

A partir d'une géométrie particulière du profil en long du type "pente et contre-pente ", l'intérêt de formules de transport "à seuil » et de lois de chargement a été démontré [4].

L'étude de l'influence de l'étendue granulométrique sur le transport et l'évolution des fonds a été effectuée au moyen d'un suivi photographique de l'interface fluide-lit et de l'analyse granulométrique de prélèvements réalisés en différents points du canal au cours du temps.

Si les essais n'ont pas réellement permis la reproduction d'un pavage statique, en revanche un tri granulométrique a clairement été mis en évidence [5].

Deux maquettes informatiques, EROS et CARICHAR [6], [7], ont été utilisées pour tester différents concepts de modélisation du transport par charriage en granulométrie étendue et tenter de reproduire les essais [8].

Il en ressort que :

l'introduction d'un facteur de masquage s'appliquant sur la force tractrice de l'écoulement ou la contrainte critique des grains, rend compte de l'exposition plus ou moins grande de certaines classes de la couche de surface,

le concept de couche mélangée opérant comme un filtre à travers lequel s'effectuent les échanges entre l'écoulement et le fond apporte une solution au problème de la modélisation de l'étendue granulométrique et du pavage.

L'ensemble des formulations du problème rendant compte de ces conclusions sont présentées dans le paragraphe suivant.

\section{Principes de la modélisation}

\subsection{Les hypothèses de la modélisation}

Le code TSAR [9] est développé sous les hypothèses suivantes :

- représentation unidimensionnelle : la seule variable d'espace est l'abscisse en long. Les autres variables sont supposées homogènes sur la section droite,

— écoulement fluvial : le nombre de Froude est limité à 0,7 ,

- faibles pentes : le code TSAR traitera des rivières de plaine dont la pente varie entre $10^{-4}$ et $10^{-3}$. Les torrents de montagne sont exclus,
- matériaux non cohésifs (sable ou gravier) en granulométrie étendue.

Le code est dévolu à l'étude de l'évolution des fonds de rivières. L'emprise des biefs traités varie de quelques centaines de mètres à quelques dizaines de kilomètres. L'échelle en temps va de quelques heures à quelques années.

La version ici décrite est limitée à des systèmes fluviaux à bief unique.

Les singularités (seuil, déversoir) n'y sont, pour l'instant, pas prises en compte.

\subsection{Représentation physique}

\subsubsection{Principaux concepts}

Le transport solide résulte, schématiquement, de l'interaction entre deux milieux :

- le fluide dont la description pourra se résumer à la connaissance en tout point et à tout instant de la cote d'eau et du débit,

- le sédiment représenté par sa densité, une discrétisation plus ou moins fine en classes granulométriques et un diamètre caractéristique pour chaque classe.

1) Hydrodynamique :

L'hydrodynamique détermine la capacité de transport en imposant la contrainte au fond. Pour tenir compte des successions de crues et de basses eaux, les équations de l'hydrodynamique seront résolues en lit mineur et en lit majeur. On en déduit la répartition des débits entre les deux lits et la valeur de la contrainte hydrodynamique totale.

\section{2) Le transport solide :}

Généralement les célérités des ondes solides étant très faibles par rapport aux célérités liquides [10] on fait l'hypothèse d'un transport saturé : la puissance érosive en un point est déterminée par les conditions hydrodynamiques locales. Exceptionnellement, on admettra un écart par rapport au transport saturé s'il existe une singularité.

Les phénomènes de charriage sont strictement limités à la zone active du lit mineur (hors berges).

Le transport solide est déterminé pour chaque classe et tient compte d'un effet de masquage : les matériaux les plus fins sont protégés par les gros. A contrario les gros matériaux sont plus exposés.

3) Couche mélangée :

L'interface liquide/solide, lieu des interactions, intervient à deux titres :

elle détermine la rugosité du lit,

elle contrôle, par sa composition, la quantité et la répartition granulométrique du charriage. L'érosion sélective de cette couche est aussi à l'origine de l'évolution des fonds vers les phénomènes de pavage.

Cette couche est communément désignée sous le nom de couche mélangée.

4) Section érodable :

La section érodable est la variable évolutive. Elle est limitée par le fond érodable et un fond inérodable (substratum rocheux, strate consolidée). Sous la couche 


\section{Notations}

A section mouillée $\left(\mathrm{m}^{2}\right)$

$A m$ section de la couche mélangée $\left(\mathrm{m}^{2}\right)$

As section érodable $\left(\mathrm{m}^{2}\right)$

$b \quad$ largeur du lit $(\mathrm{m})$

$d$ diamètre moyen du matériau

$d_{i} \quad$ diamètre moyen de la classe $i(m)$

$d_{50}$ diamètre 50 du matériau $(\mathrm{m})$

$d_{90}$ diamètre 90 du matériau $(\mathrm{m})$

diet diamètre sédimentologique diet $=d_{50}\left[(s-1) g / v^{2}\right]^{1 / 3}$

$G$ débit solide total $\left(=\sum_{k=1}^{N} G_{k}\right)\left(\mathrm{m}^{3} / \mathrm{s}\right)$

$G_{k}$ débit solide effectif de la classe $k\left(\mathrm{~m}^{3} / \mathrm{s}\right)$

$G_{k}^{*}$ débit solide potentiel de la classe $k\left(\mathrm{~m}^{3} / \mathrm{s}\right)$

$H$ hauteur d'eau (m)

$K$ coefficient de Manning Strickler global $\left(\mathrm{m}^{-1 / 3} \mathrm{~s}^{-1}\right)$

$K_{f}$ coefficient de Manning Strickler de forme $\left(\mathrm{m}^{-1 / 3} \mathrm{~s}^{-1}\right)$

$K_{q}$ coefficient de Manning Strickler de grain $\left(\mathrm{m}^{-1 / 3} \mathrm{~s}^{-1}\right)$

$K_{\text {, }}$ coefficient de la loi de chargement
$K_{\mathrm{ct}}$ coefficient de proportionnalité sur les conditions aux limites solides

p porosité

$Q$ débit liquide $\left(\mathrm{m}^{3} / \mathrm{s}\right)$

$q l$ débit d'apport linéique $\left(\mathrm{m}^{2} / \mathrm{s}\right)$

$R h$ rayon hydraulique $(\mathrm{m})$

$s$ densité du matériau

$S$ terme source solide total $\left(\mathrm{m}^{2} / \mathrm{s}\right)$

$S_{f}$ pente d'énergie

$S_{k}$ terme source solide pour la classe $k\left(\mathrm{~m}^{2} / \mathrm{s}\right)$

$t$ temps (s)

$x$ abscisse en long $(\mathrm{m})$

$\beta_{k} \quad$ pourcentage granulométrique de la classe $k$ dans la couche mélangée

$\beta_{1 k}$ pourcentage granulométrique de la classe $k$ dans la première strate

$\varepsilon^{\prime}$ coefficient d'échange entre la couche mélangée et la strate sous-jacente

$\varepsilon \quad$ coefficient de la loi de chargement

$\varepsilon=1$

ou $\varepsilon=0$

$v \quad$ viscosité de l'eau $\left(\mathrm{m}^{2} / \mathrm{s}\right)$ mélangée, la section érodable est représentée par des strates, décrites par leur épaisseur et leur composition granulométrique.

Sous l'effet de l'érosion ou du dépôt le fond érodable évolue parallèlement aux fonds initiaux.

La figure $I$ donne la représentation de la section en travers adoptée pour TSAR.

\subsubsection{Les équations}

Si $N$ est le nombre de classes granulométriques, les équations à résoudre [11], [12] sont au nombre de $N+3$ ou $2 N+3$ selon que l'on fait l'hypothèse d'un transport saturé ou non. Il s'agit d'équations de bilan sur la phase fluide et sur la phase solide :

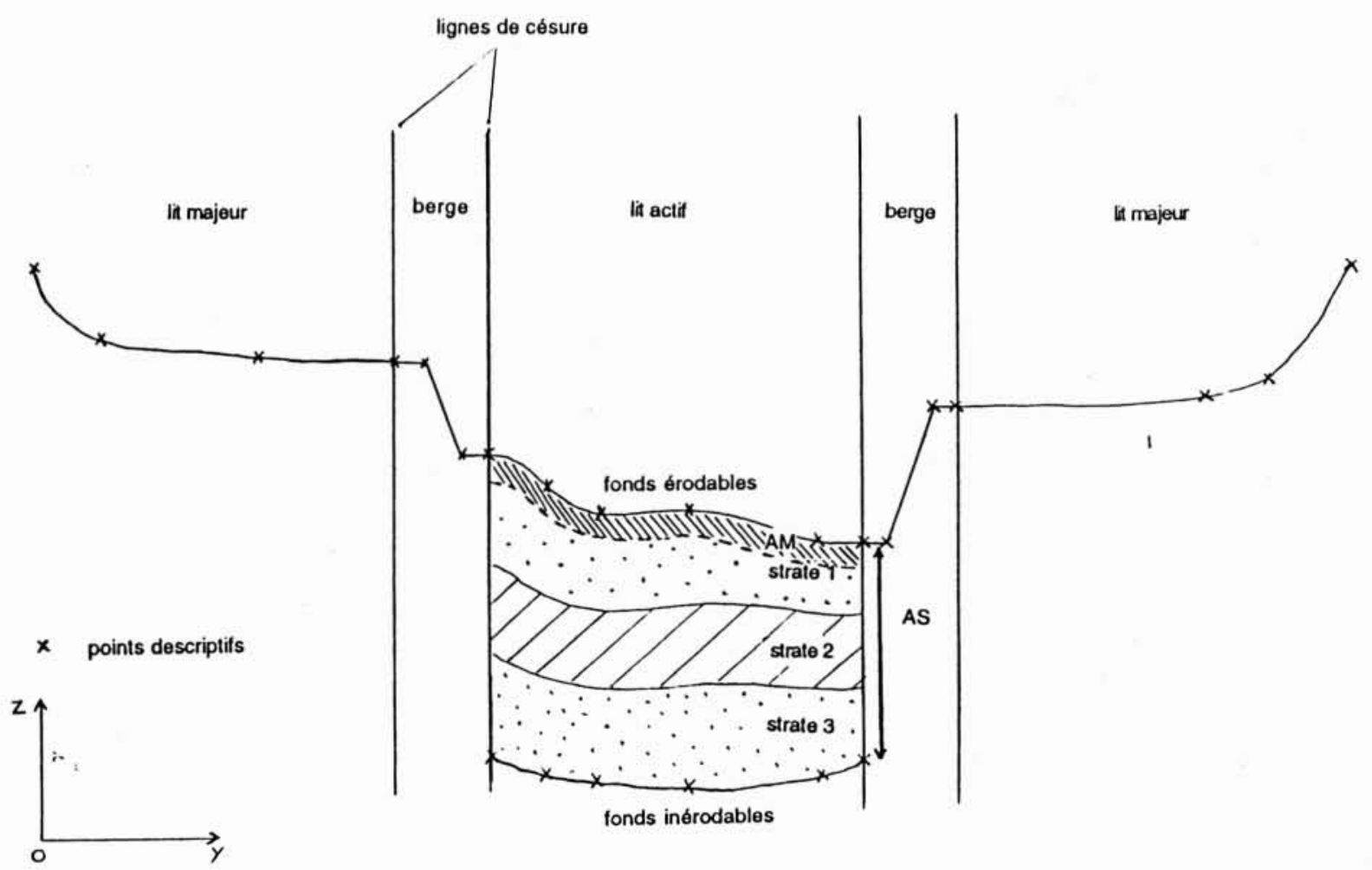

1. Représentation de la section en travers. 
Hydrodynamique : équations de Saint-Venant

Conservation de masse :

$$
\frac{\partial A}{\partial t}+\frac{\partial Q}{\partial x}=q_{1}
$$

Quantité de mouvement :

$$
\begin{aligned}
\frac{\partial Q}{\partial t}+\left(g \frac{A}{b}-\frac{Q^{2}}{A^{2}}\right) \frac{\partial A}{\partial x}+g \frac{A}{b} \frac{\partial A_{s}}{\partial x} & +2 \frac{Q}{A} \frac{\partial Q}{\partial x}= \\
& =g A \frac{A+A_{s}}{b^{2}} \frac{\partial b}{\partial x}-g A S_{f} .
\end{aligned}
$$

Solide

Conservation de masse (écrite sur l'ensemble de la section érodable) :

$$
(1-p) \frac{\partial A_{s}}{\partial t}+\frac{\partial G}{\partial x}=S .
$$

Bilans de conservation de masse pour chaque classe $k$ dans la couche mélangée :

$$
\begin{aligned}
& (1-p) \frac{\partial \beta_{k} A_{m}}{\partial t}+\frac{\partial G_{k}}{\partial x}+ \\
& +(1-p)\left[\varepsilon^{\prime} \beta_{k}+\left(1-\varepsilon^{\prime}\right) \beta_{1 k}\right]\left[\frac{\partial A_{s}}{\partial t}-\frac{\partial A_{m}}{\partial t}\right]=S_{k} .
\end{aligned}
$$

Dans le cas où l'on fait l'hypothèse d'un transport saturé : $G_{k}=G_{k}^{*}$

$\varepsilon^{\prime}=1$ s'il n'y a pas d'échange entre la couche mélangée et la première strate

$\varepsilon^{\prime}=0$ dans le cas contraire.

Lois de chargement :

Dans le cas où l'on accepte que le transport s'écarte de sa valeur à l'équilibre :

$$
\frac{\partial G_{k}}{\partial x}=K_{1}\left(G_{k}^{*}-G_{k}\right)+\varepsilon \frac{G_{k}}{G_{k}^{*}} \frac{\partial G_{k}^{*}}{\partial x}
$$

$\varepsilon=0$ loi de Daubert-Lebreton

$\varepsilon=1$ loi de Bell Sutherland.

\section{Contraintes sur le système d'équations}

Par définition, les pourcentages granulométriques doivent vérifier à tout instant et en tout point :

$$
\sum_{k=1}^{N} \beta_{k}=1
$$

Or la somme des $N$ équations de bilan de classe, s'écrit, compte tenu de la continuité solide :

$$
\frac{\delta\left(\sum_{k=1}^{N} \beta_{k}\right)}{\partial t}=0 .
$$

La contrainte sera donc vérifiée si :

la discrétisation des équations est cohérente,

la contrainte est vérifiée au premier pas de temps.

La deuxième contrainte porte sur le signe des pourcentages granulométriques :

$$
\beta_{k} \geqslant 0, \quad \forall k .
$$

\section{Couplage et non-linéarités du système d'équations}

Physiquement, le liquide et le solide sont couplés. Cela se traduit dans les équations par le terme $A_{s}$ qui intervient dans les équations de Saint-Venant d'une part et dans le calcul de $G_{k}^{*}$ à partir de la contrainte hydrodynamique d'autre part. En pratique, compte tenu de la grande différence des célérités entre les ondes liquides et solides, les équations pourront être traitées séparément au prix du respect de certains critères de stabilité et de précision (découplage numérique).

Les non-linéarités du système apparaissent à plusieurs niveaux :

- termes croisés, notamment dans les équations de bilans des classes où $A m$ est fonction des inconnues $\beta_{k}$ (voir $\$ 3.3 .3$ ):

- calcul de $G_{k}{ }^{*}$, où le facteur de masquage intervient et qui est, lui aussi, fonction des $G_{k}^{*}$ par l'intermédiaire du diamètre médian de la classe.

\subsection{Fermeture}

\subsubsection{Rugosité}

La rugosité du lit $K$, est exprimée par le coefficient de Manning-Strickler. Seule la rugosité du lit actif est susceptible de variations : elle est fonction des rugosités de grain $K_{g}$ et de forme $K_{f}$.

La rugosité de grain représente la rugosité des lits plats. Elle est liée par l'ensemble des auteurs à un diamètre caractéristique des grains.

La rugosité de forme représente la rugosité liée à la présence sur le fond de rides ou dunes. Son expression varie selon les auteurs.

Deux approches sont retenues dans TSAR :

Approche de Ramette/Chollet-Cunge [13]

Rugosité de grain :

$$
K_{g}=\frac{26}{d_{90}^{1 / 6}} .
$$

Rugosité de forme :

la formule de Ramette est fondée sur des essais réalisés par Engelund HANSEN. La formule a été modifiée pour mieux prendre en compte les essais de laboratoire où rayon hydraulique et tirant d'eau sont nettement distincts. Il vient alors :

$$
K_{f}=11,3 \sqrt{g d_{50}} R_{h}^{-2 / 3}\left[0,06+0,16\left(\frac{H J}{d_{50}}\right)^{2}\right]^{5 / 8} J^{-5 / 8}
$$

avec

$$
J=J_{e}-\frac{Q^{2}}{g\left(b+\frac{H}{3}\right)^{3} H^{3}}\left[\left(b+\frac{2 H}{3}\right) J_{e}-b J_{s}\right]
$$

$J_{e}:$ pente de la ligne d'eau,

$J_{s}:$ pente des fonds.

Dans TSAR, cette approche est associée aux formules de transport de Meyer Peter Muller et Engelund Hansen.

La rugosité résultante du lit actif est le minimum des rugosités de grain et de forme. 
Approche de Van Rijn [8]

VAN RIJN utilise le coefficient de Nikuradse. En notant alors $k_{s c}^{\prime}$, la rugosité de grain et $k_{s c}^{\prime \prime}$, la rugosité de forme, la rugosité résultante est :

$$
k_{s}=k_{s c}^{\prime}+k_{s c}^{\prime \prime} .
$$

La rugosité de grain $k_{s c}^{\prime}=3 d_{90}$.

La rugosité de forme est estimée par VAN RIJN à partir des caractéristiques des dunes ou rides.

\subsubsection{Transport solide}

Trois lois de transport solide ont été retenues dans le cadre de TSAR. Elles sont exprimées ci-dessous en $\mathrm{m}^{3} / \mathrm{s}$.

Meyer Peter MULLER :

$$
G=8 \sqrt{(s-1) g d^{3}} b\left[\mu \tau *-\xi \tau_{c}^{*}\right]^{3 / 2} .
$$

Engelund HANSEN :

$$
G=0,05 K^{2} R_{h}^{1 / 3} \sqrt{\frac{(s-1) d^{3}}{g}} \tau_{*}^{5 / 2} b .
$$

\section{VAN RIJN :}

$$
G=0,053 \sqrt{g(s-1)} \frac{d^{1,5}}{\operatorname{diet}^{0,3}} b\left(\mu \frac{\tau}{\tau_{c}}-\xi\right)^{2.1} .
$$

\section{Remarques}

Le coefficient de masquage $\xi$ qui intervient dans les । formules de Van Rijn et Meyer Peter Muller est donné, dans TSAR, par la formule d'Egiazaroff, pour chaque classe $k$ :

$$
\xi_{k}=\left[\frac{\log (19)}{\log \left(19 \frac{d_{k}}{d}\right)}\right]^{2}
$$

$\mu$ est le rapport entre la contrainte efficace (explicative du transport) et la contrainte hydrodynamique totale.

Pour Meyer Peter Muller $\quad \mu=\frac{Q_{\text {char }}}{Q}\left(\frac{K}{K_{g}}\right)^{3 / 2}$

$Q_{\text {char }}$ est le débit de charriage. En première approximation, on retient $Q_{\text {char }}=Q$.

Pour VAN RIJN $\quad \mu=\left(\frac{K}{K_{g}}\right)^{2}$.

\subsubsection{Couche mélangée}

L'évolution des pourcentages granulométriques de chaque classe dans la couche mélangée est donnée par les équations de bilan des classes. Il reste donc à déterminer la surface ou l'épaisseur de la couche mélangée pour « fermer» le problème.

La détermination de cette variable doit représenter le mieux possible la physique de phénomène et éviter les ennuis numériques.

La couche mélangée contient les matériaux susceptibles d'être érodés ou déposés en fonction de l'évolution de la contrainte hydrodynamique. Physiquement son épaisseur est donc liée à la puissance érosive du fluide mais aussi à la composition même de la couche en contact avec le fluide. Le cas extrême est le cas de pavage où l'épaisseur de cette couche est directement liée à la taille des plus gros matériaux en présence.

De façon pragmatique, l'épaisseur de la couche mélangée varie entre deux valeurs extrêmes:

l'épaisseur de Borah : $\quad H_{b}=\frac{1}{1-p} \frac{d_{L}}{\sum_{k=L}^{N} \beta_{k}}$

$d_{L}$ : diamètre de la plus petite classe non mobilisable

la hauteur des dunes: $H_{d}=C_{d} . H$

$C_{d}$ constante de l'ordre de 0,1 à 0,2 .

\subsection{Le mode numérique}

Le schéma numérique retenu pour la résolution du système d'équations est un schéma explicite itératif aux différences finies.

Les équations du fluide et du solide sont résolues séparément. Toutefois, des itérations assurent le couplage physique et permettent de vérifier la contrainte

$$
\sum_{k=1}^{N} \beta_{k}=1
$$

Les différences finies sont écrites selon un schéma de Preissmann :

$$
\begin{aligned}
\begin{aligned}
\frac{\partial T}{\partial x}=(1-\psi)\left(\frac{\partial T}{\partial x}\right)_{i} & + \\
& +\theta\left(\frac{\partial T}{\partial x}\right)^{n+1} n \text { indice de temps } \\
\frac{\partial T}{\partial t}=(1-\psi)\left(\frac{\partial T}{\partial t}\right)_{i}+ & \\
& +\psi\left(\frac{\partial T}{\partial t}\right)_{i+1} i \text { indice despace. }
\end{aligned}
\end{aligned}
$$

Pour la version itérative du schéma explicite, il n'existe pas de résultat théorique sur la convergence du schéma. Il est possible de reprendre la limite sur le pas de temps imposée par le nombre de courant $\mathrm{C} 1$ calculé sur les célérités solides pour $\theta=1$ [14], [15]:

$$
C 1 \frac{\delta t}{\delta x}<2 \psi-1 \text {. }
$$

Les premiers tests sur les valeurs de $\theta$ et $\psi$ nous ont fait choisir $\theta=0,5$ et $\psi=1$. Ces valeurs sont utilisées lors des cas tests suivants.

Un organigramme schématique est porté sur la figure 2.

Le code est programmé en Fortran. Les équations du solide sont calculées en double précision.

\section{Tests comportementaux}

Le code a fait l'objet de plusieurs tests pour vérifier la qualité des résultats et la robustesse du schéma.

Nous présentons ci-dessous deux cas tests comportementaux : 


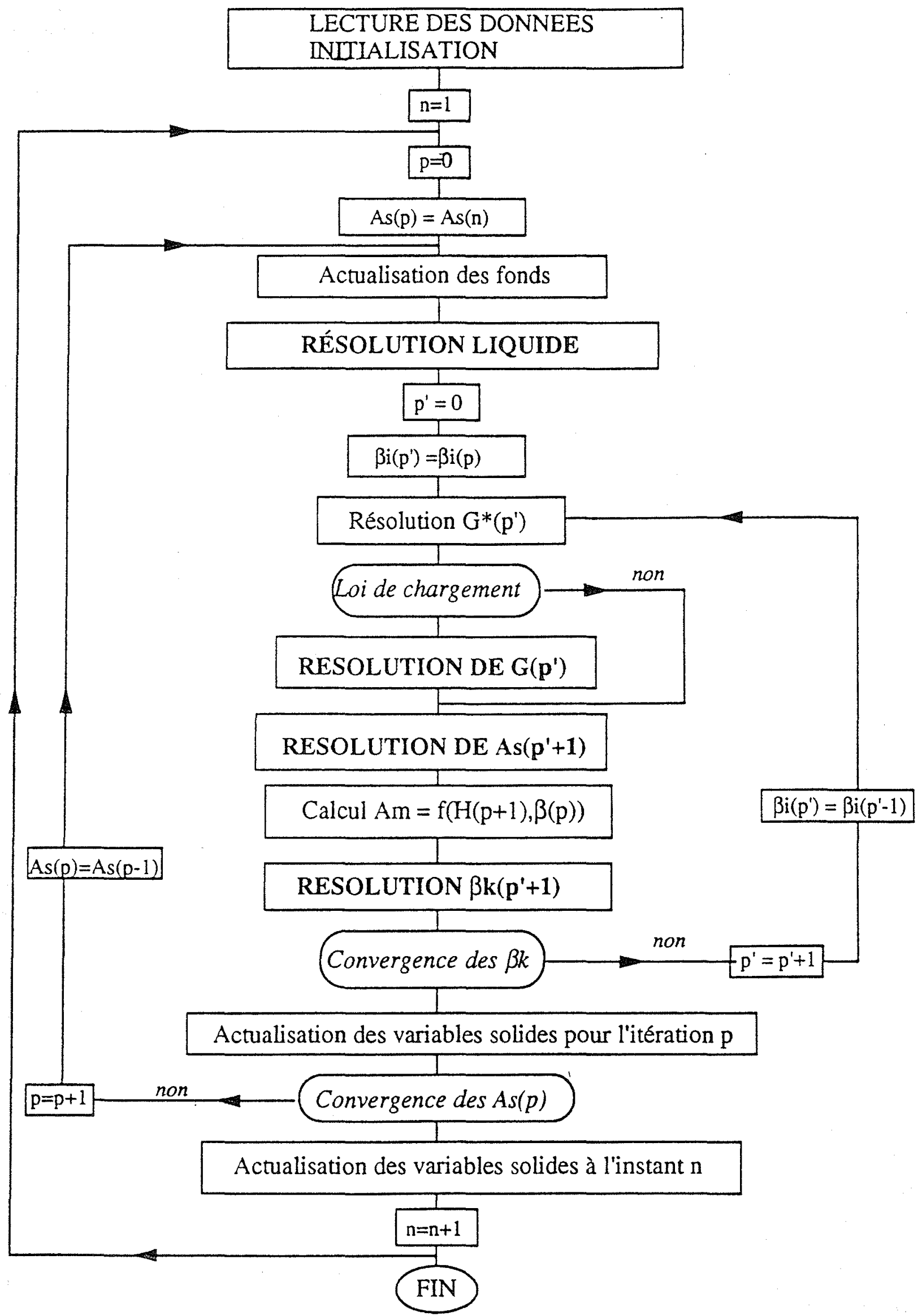

2. Organigramme de la méthode explicite itérative. 
bief limité par un barrage à l'amont: on étudie la fosse d'érosion liée au déficit en matériau à l'aval immédiat du barrage ;

bief limité par un barrage à l'aval. Le barrage arrête les sédiments et on étudie le dépôt à l'amont immédiat du barrage.

\subsection{Barrage à l'amont}

Le cas test représente un bief de $20 \mathrm{~km}$ de long en lit mineur exclusivement, dont la section en travers est trapézoïdale (fig. 3).

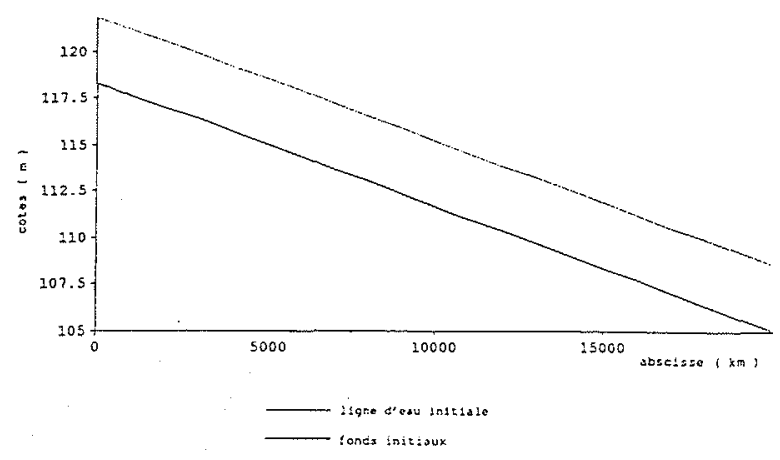

CONDTTIONS INTTTALES

PROFIL EN TRAVERS

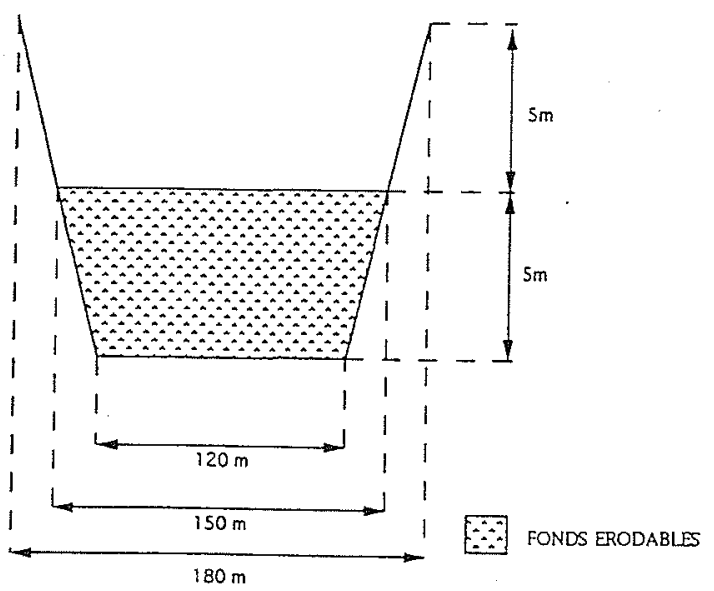

3. Test barrage amont : conditions initiales et profil en travers.

Les sections de calcul sont espacées de $100 \mathrm{~m}$ sur le premier kilomètre, puis de $1 \mathrm{~km}$ jusqu'à l'aval.

La durée de simulation est de 35 jours avec un pas de temps de $6 \mathrm{~h}$.

Le sédiment est représenté par une classe granulométrique de diamètre $5 \mathrm{~mm}$.

La loi de transport solide utilisée est celle de Meyer Peter Muller. La loi de chargement est celle de Daubert Lebreton avec $K_{1}=0,01$ et $K_{1}=0,001$ (fig. 4).

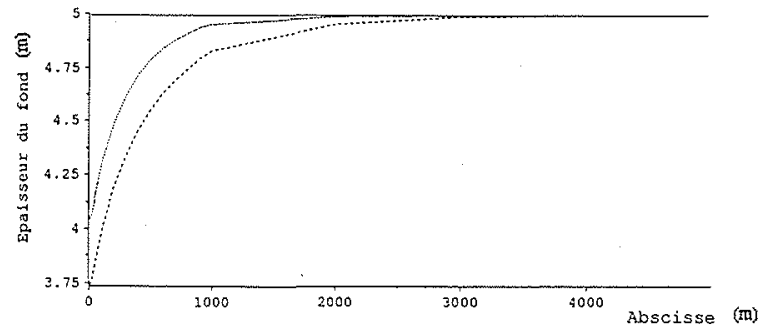

a - Coefficients de proportionnalite et de la loi de chargement $=0.01$

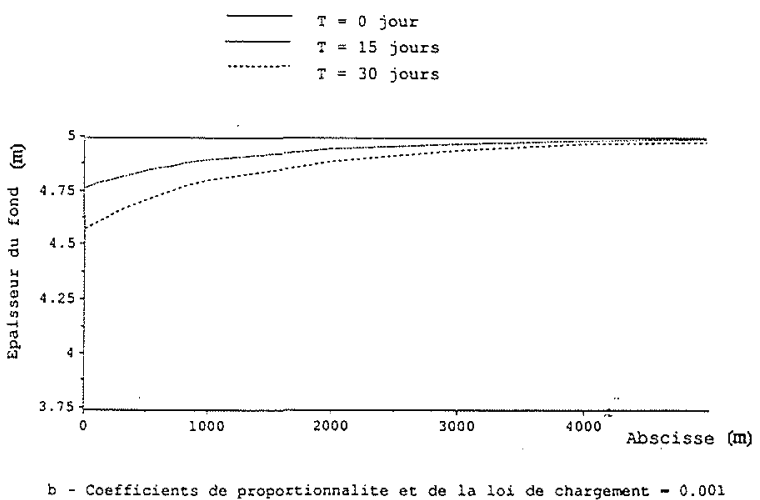

4. Test barrage amont: épaisseurs des fonds à différents instants et powr différentes valeurs de coefficients de proportionnalité et de loi de chargement.

La couche mélangée a été supposée constante, égale à $0,3 \mathrm{~m}$.

Le coefficient de Strickler est constant, égal à 30 , pendant toute la durée de l'essai

\section{Conditions alux limites}

liquide : débit amont imposé égal à $1000 \mathrm{~m}^{3} / \mathrm{s}$, cote aval imposée constante, la figure 3 représente la ligne d'eau initiale ;

solide : débit solide amont nul, la section solide amont est calculée en fonction de l'écart entre débit solide potentiel et débit solide effectif au point amont. C'est cet écart qui est responsable de l'érosion à l'aval du barrage. L'équation de continuité solide en ce point s'écrit :

$$
\frac{\partial A_{s}}{\partial t}+\frac{K_{c l}}{1-p}\left(G_{1}^{*}-G_{1}\right)=0 .
$$

Le coefficient de proportionnalité $K_{r l}$ a donc une influence considérable sur la valeur de la section érodable au point 1 .

Cette expression étant obtenue en remplaçant dans l'équation de bilan le gradient longitudinal de débit solide par sa valeur selon la loi de chargement [16], il vient $K_{c l}=K_{\mathrm{I}}$.

\section{Principales conclusions}

La figure 4 donne les profils spatiaux de l'épaisseur des fonds à différents instants (instant initial, 15 jours, 30 jours) pour deux valeurs du coefficient de la loi de chargement $K_{1}\left(K_{c l}=K_{1}\right)$. 
De façon qualitative, on retrouve la fosse d'érosion à l'aval immédiat du barrage :

la profondeur de la fosse varie avec $K_{c l}$ conformément à la condition à la limite amont ;

l'influence de l'érosion vers l'aval est d'autant plus importante que le coefficient de la loi de chargement diminue.

La discrétisation des équations aux points 1 et 2 montrent que la forme de la fosse d'érosion dépend de la valeur de $K_{1}$ mais aussi du coefficient $\psi$. Des tests supplémentaires de sensibilité doivent alors porter sur les valeurs de ces coefficients qui ne pourront être vraiment calés que sur cas réels.

Le pas de temps, dont la valeur a varié entre $900 \mathrm{~s}$ et $6 \mathrm{~h}$, n'influence pas les résultats.

La convergence est assurée en une itération pour le liquide comme pour le solide.

\subsection{Barrage à l'aval}

Le cas test représente un bief de $20 \mathrm{~km}$ de long, avec lits mineur et majeur, dont la section en travers est trapézoïdale (fig. 5).

Les sections de calcul sont espacées de $1 \mathrm{~km}$.

La durée de simulation est de 35 jours avec un pas de temps de $6 \mathrm{~h}$.

Un barrage à l'aval arrête les sédiments : le transport solide s'annule.

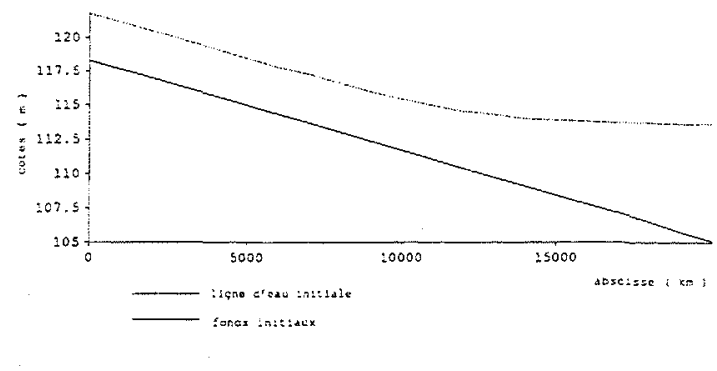

CONDTIONS INYTIALES ET EMMTES
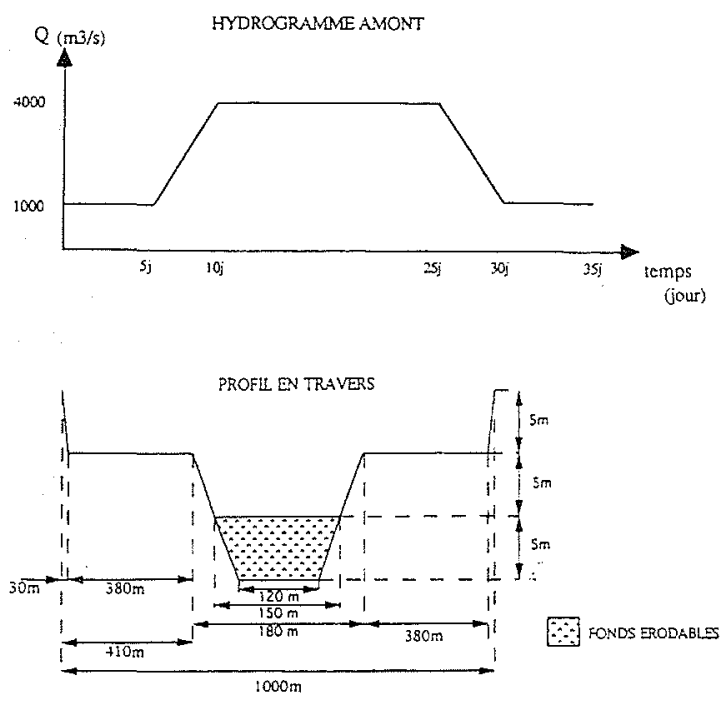

5. Test barrage aval.
Le sédiment est représenté par trois classes granulométriques de caractéristiques suivantes:

\begin{tabular}{|c|c|c|}
\hline Classes & $\begin{array}{c}\text { Diamètres limites } \\
(\mathrm{m})\end{array}$ & $\begin{array}{c}\text { Pourcentages } \\
\text { granulométriques } \\
\text { initiaux }\end{array}$ \\
\hline 1 & $0,001-0,003$ & 0,35 \\
\hline 2 & $0,003-0,005$ & 0,30 \\
\hline 3 & $0,005-0,010$ & 0,35 \\
\hline
\end{tabular}

La loi de transport utilisée est celle de Meyer Peter Muller sans loi de chargement.

La couche mélangée a été supposée constante, égale à $0,3 \mathrm{~m}$.

Le coefficient de Strickler est constant, égal à 30 , pendant toute la durée de l'essai.

\section{Conditions aux limites}

liquide : débit amont imposé selon un hydrogramme donné en figure 5 , cote aval imposée constante;

solide: débit solide amont égal au débit à saturation, section solide amont imposée constante, pourcentages granulométriques calculés en fonction de l'écart entre débits solides potentiels et débits solides effectifs au point amont. Dans ce cas particulier, cela revient à imposer une valeur constante des pourcentages granulométriques.

\section{Principales conclusions}

La figure 6 donne l'évolution de l'épaisseur du fond (c. à-d. épaisseur maximale entre les fonds inérodables et l'interface fluide/solide) ainsi que l'évolution des pourcentages granulométriques sur l'avant dernière section.

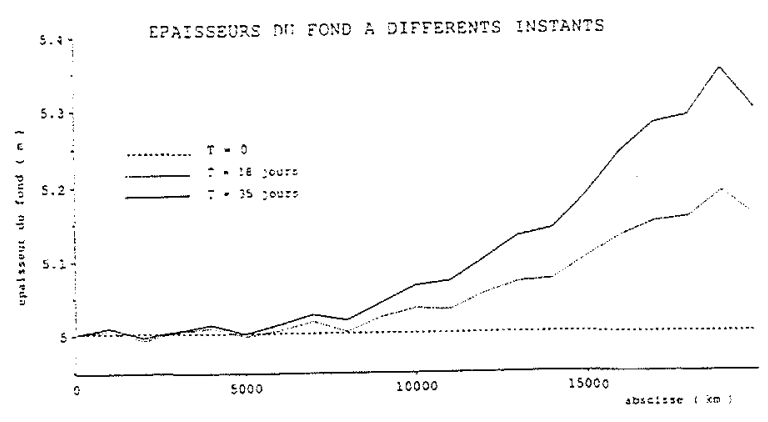

EVQLUTZON DES POURCENTAGES GRANULOMETRTQUES

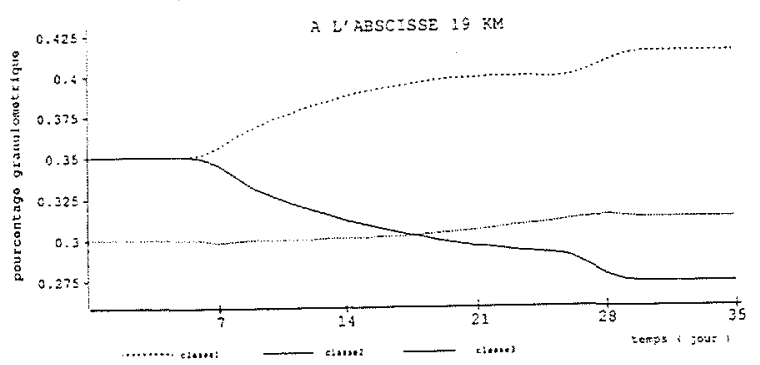

6. Test barrage aval : évolution des variables solides. 


\section{SIMULATION DES ÉVOLUTIONS DES FONDS ALLUVIONNAIRES}

L'épaisseur des fonds traduit correctement le dépôt à l'amont immédiat du barrage. Les quelques « oscillations » à l'amont du bief sont dues exclusivement à des fluctuations sur le tirant d'eau, non significatives sur les résultats hydrauliques, mais amplifiées sur le transport solide. Elles ne s'amplifient nullement en fonction du temps.

L'évolution des pourcentages granulométriques pour chaque classe montre essentiellement l'augmentation dans la couche mélangée de la classe 1, transportée depuis l'amont, et la diminution du taux de présence de la classe 3 moins mobile.

Ces résultats qualitatifs vont bien dans le sens physique.

Les pas de temps testés ont varié entre $900 \mathrm{~s}$ et $6 \mathrm{~h}$ et n'influencent pas les résultats.

La convergence des résultats est assurée en 2 itérations sur les variables solides et une seule sur le liquide.

\section{Conclusions et perspectives}

La version charriage du code TSAR, présentée ci-dessus, a été testée sur quelques cas comportementaux. Bien que de nombreux cas de validation restent à traiter, notamment pour tester l'ensemble des options disponibles (couche mélangée variable, rugosité variable, types de conditions aux limites), les premiers résultats sont très encourageants du point de vue qualitatif, mais aussi du point de vue numérique.

La méthode explicite itérative a montré que l'on représentait bien le couplage physique entre les équations et que la convergence sur le type de cas tests étudiés était rapide.

\section{Références}

[1] Rovas G., Monadier P. - La modélisation du transport solide en rivière: une action à caractère national. La Houille Blanche 3/4, 1989, pp. 259, 262.

[2] Allard J. - Modélisation du transport solide en rivière. Canal 24 : essais en régime permanent et en granulométrie uniforme. Laboratoire National d'Hydraulique, 1986.
[3] Allard J, Chée P., Foulon G. - Modélisation du transport solide en rivière. Canal 24 : essais en régime permanent pour des granulométries uniformes et non uniformes. Laboratoire National d'Hydraulique, 1988.

[4] CHÉE P., KIEFFER D. - Essai 56. Contre-pente et seuil fixe. Sable: de 4 à $5 \mathrm{~mm}$. Débit amont $=20 \mathrm{ls}$. Laboratoire National d'Hydraulique, 1989.

[5] Ben Slama E., Chée P., KiefFer D. - Essai 58. Contrepente et seuil mobile à l'aval. Sable : de 1 à $10 \mathrm{~mm}$. Débit amont $=25 \mathrm{l} / \mathrm{s}$. Laboratoire National d'Hydraulique, 1990.

[6] Rahuel J.L., Belleudy Ph. - La simulation numérique des phénomènes de tri granulométrique. La Houille Blanche 3/4, 1989, pp. 268-272.

[7] Rahuel J.L., Chollet J.P. - Modèles d'évolution des lits alluvionnaires soumis à charriage : tri granulométrique et pavage. La Houille Blanche 3/4, 1989, pp. 273-276.

[8] Projet TSAR, Note C. Formules de transport par charriage. EDF/LNH-LHF, 1989.

[9] Spécifications et conception détaillée du code TSAR. EDF/LNH-LHF, novembre 1990

[10] Cunge J.A., Holly F.M., Verwey A. - Practical aspects of computationnal river hydraulics. Iowa Institute of hydraulics research, 1980, Univ, of Iowa, lowa city IA 52242.

[11] Holly F.M., Rahuel J.L. - Nowveau cadre numéricophysique de modélisation des rivières à fonds mobiles. Ire Partie : Principes numériques er physiques. J. of Hydraulic Research, Vol. 28, 1990, n ${ }^{\circ}$ 4, AIRH

[12] Belleudy Ph., SchüttrumpF H. - Modélisation de l'évolution morphologique du Danube et de l'Isar. Journées transport solide, SHF, Paris, novembre 1993.

[13] Chollet J.P., Cunge J.A. - New interpretation of some head loss-flow velocity relationships for deformable movable beds. J. of Hydraulic Research, Vol. 117, 1979, n' 1, AIRH.

[14] VAN RUN L.C. - Handbook sediment transport by currents and waves. Delft Hydraulics, 1989.

[15] Gousseballe J., Labadie G., Rahuel J.L. - Proje TSAR, Note G. Aspects numériques. EDF/LNH-LHF, 1989

[16] LrN, GoodwIN. - Stability of a general Preissmam scheme. Journal of Hydraulic Engineering, 1987, Vol. 113 , $\mathrm{N}^{\circ} 1$, pp. 16 à 28 .

[17] Rahuel J.L. - Modélisation de I'évolution du lit des rivières allwionnaires à granulométrie étendue. Thèse de l'Institut National Polytechnique de Grenoble, 1988. 